\title{
Method and Device for Reducing the Toxicity of Diesel Engine Exhaust Gases
}

\author{
Byshov N.V. ${ }^{1}$, Bachurin A.N. ${ }^{2}$, Bogdanchikov I.Yu. ${ }^{3}$, Oleynik D.O. ${ }^{4}$, Yakunin Yu.V. ${ }^{5}$, Nelidkin A.V. ${ }^{6}$ \\ ${ }^{I}$ Doctor of Technical Science, Professor, Ryazan State Agrotechnological University Named after P.A. Kosychev, \\ ${ }^{2}$ Candidate of Technical Science, Associate Professor, Head of the Faculty of Maintenance of Machine and Tractor Fleet, Ryazan State \\ Agrotechnological University Named after P.A. Kosychev, \\ ${ }^{3}$ Candidate of Technical Science, Associate Professor of the Faculty of Maintenance of Machine and Tractor Fleet, Ryazan State \\ Agrotechnological University Named after P.A. Kosychev, \\ ${ }^{4}$ Candidate of Technical Science, Associate Professor, Associate Professor of the Faculty of Maintenance of Machine and Tractor Fleet, \\ Ryazan State Agrotechnological University Named after P.A. Kosychev, \\ ${ }^{5}$ Senior Teacher of the Faculty of Maintenance of Machine and Tractor Fleet, Ryazan State Agrotechnological University Named after \\ P.A. Kosychev, \\ ${ }^{6}$ Postgraduate, Ryazan State Agrotechnological University Named after P.A. Kosychev
}

\begin{abstract}
The aim of the article is to develop a method and a device for reducing the toxicity of exhaust gases of diesel engines and reducing noise taking into account the current mode of operation of the engine. This is done with the help of installing a liquid catalyst (LC) into the exhaust system, ensuring the processes of trapping, chemical bonding and neutralization of toxic components and soot particles in the aerosol chamber while the vortex flow is being processed by a neutralizing solution supplied under pressure. Then the flow is divided into phases and toxic components and soot are separated in the centrifugal swirl drop separator (SDS).

The developed and tested design of an exhaust gas cleaning device installed instead of the standard D-120 engine exhaust system and an automated cleaning process control system make it possible to reduce the toxicity of exhaust gases (EG): nitrogen oxides by $40 \%$, hydrocarbons by $43 \%$ and soot by $70 \%$. The noise level of its work in enclosed spaces was reduced by $16-22 \%$. The device also had low gas-dynamic resistance.

The investigation methodology is based on the use of modern methods and measuring devices. Exhaust gas tester META "Autotest CO $\mathrm{CH}-\mathrm{CO}_{2}-\mathrm{O}_{2}-\lambda-\mathrm{NO}_{\mathrm{x}}$ " was used to measure the toxicity of exhaust gases. To measure smoking at the exhaust of the diesel engine, the opacity meter META-01MP was used. The gas flow velocity was measured with ATT-1004 thermo-anemometer, the noise level of the tractor was recorded with noise and vibration meter VSHV-003-M2, and the fuel consumption with SIRT-1 meter.

Theoretical studies were carried out on the basis of the laws of gas dynamics, the modern theory of statistical analysis, and experiment planning techniques. When developing an experimental LC model, dependencies were obtained, which allow to achieve the optimal design and technological parameters of the wet cleaning system for diesel exhaust gases.

The optimization of the design parameters and the processing of experimental data were carried out with the help of modern software using the methods of mathematical statistics using computers.

The current methods of reducing the toxicity of engines consist primarily in improving the design of engines, in order to influence the nature of the working process, the use of alternative fuels and additives, exhaust gas recirculation, as well as installing various types of exhaust gas catalytic systems. Measures related to the introduction of constructive changes in engines require some major res tructuring of the industry, which is difficult to achieve in modern conditions. Alternative fuels have not yet been widely used in agriculture. Therefore, today the most effective and acceptable means of achieving environmental standards is the installation of various mobile catalysts in the exhaust system, as well as devices for trapping soot particles. The use of this exhaust gas cleaning system for diesel engines functioning in enclosed spaces can significantly improve the working conditions of the personnel and have a slight effect on the power and fuel-economic performance of the power unit, reducing the power of the D-120 engine of the T-30 tractor equipped with an upgraded exhaust system when taking external speed characteristics averaged $1.6 \%$, the torque was $1.5 \%$ and the increase in specific fuel consumption was $1.8 \%$.
\end{abstract}

In this paper we used materials from scientific publications indexed by bibliographic abstract databases of Scopus and Web of Science.

Keywords: toxicity, diesel engines, exhaust gas cleaning, catalytic conversion of toxic components, liquid catalyst (LC), nitrogen oxides, soot.

\section{Introduction}

Improving environmental safety in the agro-industrial complex is a priority for our country. This is due to the transition of the country to the European environmental standards set for emissions of harmful substances with exhaust gases. Mobile power tools pollute the air with exhaust gases from diesel engines, crankcase gases and vapor of fuels used in the process of working equipment. According to statistical data [11], 463.6 thousand units of automotive equipment, 125.8 thousand combines and 132.3 
thousand units of other equipment (beet harvesting machines, sprinklers and watering machines, solid mineral fertilizers, etc.) are currently used in the Russian Federation. Due to the deterioration of the technical state of machines, the ecological situation also changes dramatically. The machines, released more than 30 years ago, are still in operation and, of course, they do not meet modern environmental requirements. During the combustion of $1 \mathrm{~kg}$ of diesel fuel, up to $100 \mathrm{~g}$ of toxic components are released into the environment. According to experts, the total mass of emissions of all diesel engines in operation in the CIS countries averages 15 million tons per year [11].

Currently, mobile energy vehicles, carrying different technological equipment, are widely used at enterprises of the agro-industrial complex. At the same time, as a power unit, they are usually equipped with diesel engines, which are less toxic and more efficient as compared to gasoline counterparts [24].

The content of toxic substances in the air overwhelms the health of workers and animals, and adversely affects the development of agricultural crops, thereby reducing the quality of agricultural products and the service life of buildings and equipment $[29,30$, $31,32]$.

To eliminate this factor, it is necessary to reduce the toxicity of exhaust gases (EG) of the diesel engine to the permissible limits while maintaining the proportion of engineering work.

Therefore, the purpose of this study is to develop a method and device for reducing exhaust emissions of diesel engines and noise by installing a liquid catalyst (LC) into the exhaust system, ensuring the processes of trapping, chemical bonding and neutralization of toxic components and soot particles in the aerosol chamber while the vortex flow is being processed by a neutralizing solution supplied under pressure. Then the flow is divided into phases and toxic components and soot are separated in the centrifugal swirl drop separator (SDS).

The application of this method will not require making any significant changes in the design of the diesel exhaust system and will maintain the overall dimensions of the mobile power unit.

\section{Material Sand Methods}

We have evaluated the impact of mobile energy units on the conditions of employment and the quality of products. EG emission is a consequence and a necessary condition for the normal functioning of the internal combustion engine. The engine produces mechanical energy by oxidizing the fuel with air, carrying out heat and mass exchange with the environment during operation. The EG of diesel engines is a complex multicomponent gas mixture. Our theoretical studies show that EGs contain more than 1000 different substances. For most components of the EG, maximum allowable concentrations (MACs) are established. They are determined on the basis of the principle of the complete absence of their negative impact on humans.

The current state of work to reduce the EGs toxicity and opacity of functioning diesel engines is characterized by the fact that at the moment there is no single universal tool that provides a fairly complete reduction of harmful engine emissions. We have analyzed modern methods and means of reducing the toxicity of exhaust gases of engines $[1,2,3,4,5,6,7,8,9,10]$.

The analysis of existing designs of devices for wet cleaning of EGs of the internal combustion engine revealed a number of problems that have a significant impact on both the efficiency of EG cleaning and the possibility of their use in general $[12,15,23]$. The most significant of them are as it follows:

- large dimensions and weight of devices;

- the need for frequent change of the working solution or water;

- a sharp decrease in the efficiency of neutralization when the engine is running at modes close to nominal;

- large hydraulic resistance.
In order to solve these problems, the designs of devices $[16,17$, $20,21,22,25,26]$ for cleaning EGs of diesel internal combustion engines were developed. The scheme of one of them is presented in Figure 1.

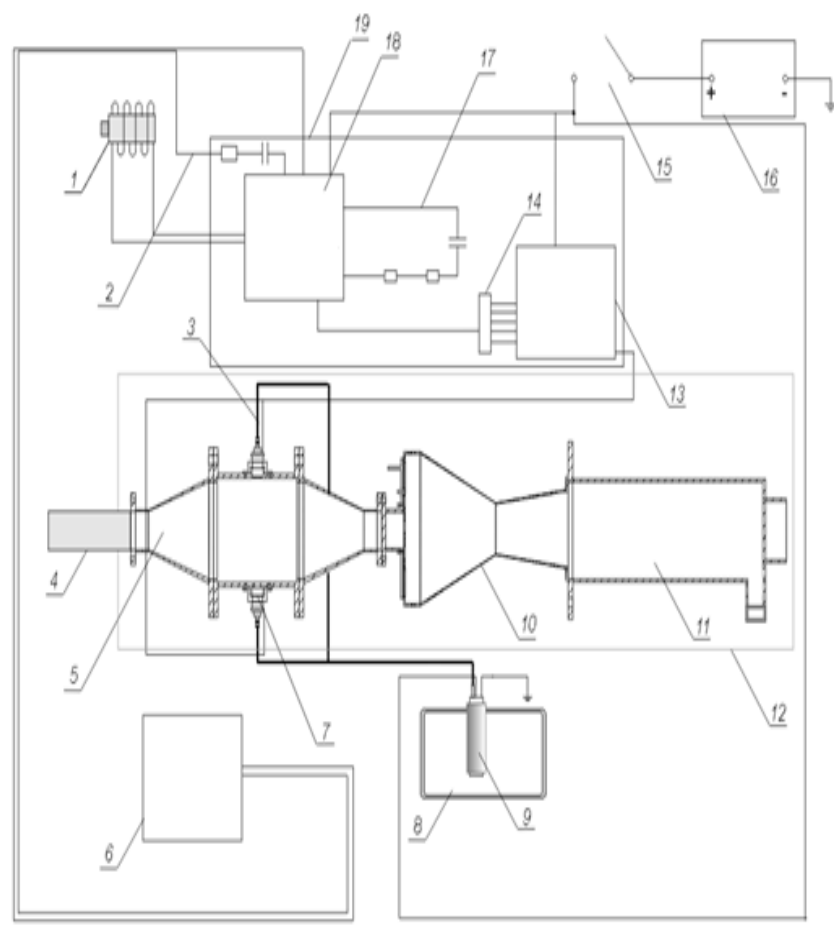

1 - crankshaft position sensor (CPS); 2, 17 - time-setting circuits; 3- metal tubes for supplying a neutralizing solution; 4 - exhaust manifold; 5 aerosol chamber; 6 - regulator position sensor (RPS); 7 - nozzles; 8 - tank with a neutralizing solution; 9 - liquid pump; 10 - ejector; 11 - swirl drop separator; 12 - liquid catalyst (LC); 13 - key block; 14 - resistor assembly; 15 - key; 16 -power supply; 18 - integral timer unit; 19 - electronic control unit.

Figure 1: Diagram of the device for cleaning the EGs of diesel engines.

The types explaining the device of the LC and the technology of its work are presented, respectively, in Figures 2 and 3.

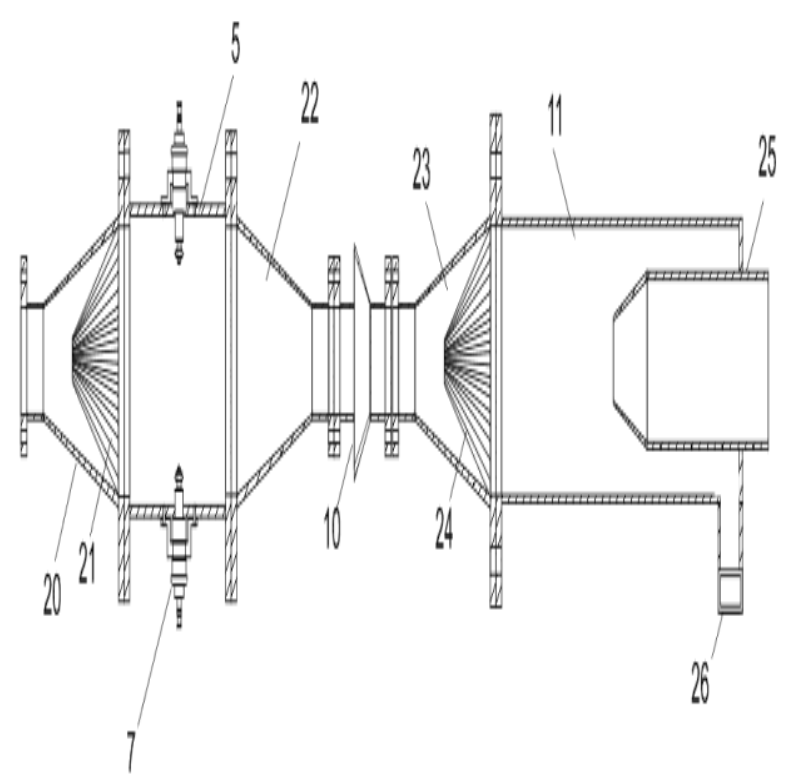

20 - aerosol chamber inlet; 21,24 - conical swirl; 22 - aerosol chamber outlet; 23 - inlet of the centrifugal swirl drop separator; 25 - outlet of the centrifugal swirl drop separator; 26 - fluid removal pipe.

Figure 2: Diagram of the EG liquid catalyst. 


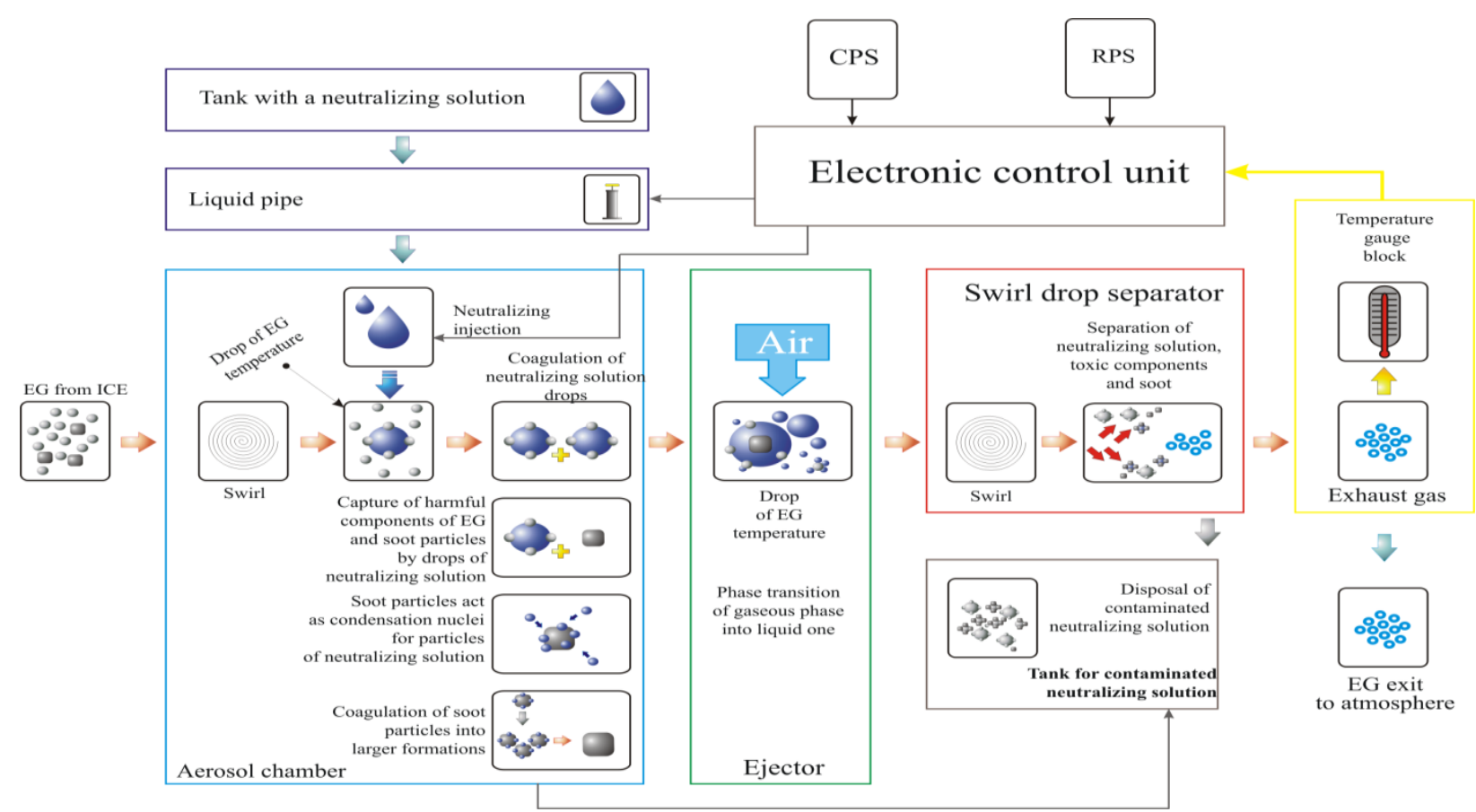

Figure 3: Diagram of the diesel engine EG cleaning by the device.

The electronic control unit (ECU) of the LC(No. 19,Fig. 1)is to manage the supply of the neutralizing solution to aerosol chamber 5 by nozzles 7 . ECU 19 is connected to the vehicle's on-board network with a nominal $12 \mathrm{~V}$ DC. It has connectors for crankshaft position sensor 1 , regulation position sensor 6 , temperature gauge, four nozzles 7 and power supply 16 .

The main element of electronic control unit 19 is an IC timer-chip "NE555" series, generating an electrical signal of a given duration. IC timer-chip "NE555" is a stable controller capable of generating accurate time delays or periodic oscillatory signals. In the case when the chip functions in the delay generation mode, their duration is precisely set using one external resistor and one capacitor. When operating as a generator of periodic oscillations, the frequency and duty ratio of a signal are very accurately specified by one external capacitor and two external resistors.

To make the ECU work, two time-setting circuits 2 and 17 are connected to the microchip. The first time-setting circuit containing external capacitors of fixed capacity of the K73-9 type and a resistor regulates the time of the nozzle in the open state, i.e. aerosol injection time. Element values are selected in such a way that the duration of the electrical signal is $15 \mathrm{~ms}$. The second timesetting circuit containing one external capacitor and two external resistors sets the time of the nozzle being closed, i.e. it determines the frequency of the aerosol injection for test mode. This characteristic is set using constant resistances. Idle time will be equal to $20 \mathrm{~ms}$. In a case variable resistance in the circuit, it becomes possible to adjust the frequency of the aerosol injection to the engine modes of operation smoothly. For this purpose, the circuit includes crankshaft position sensors and a lever of the allrange regulator of the fuel pump. In this case, the frequency of the aerosol injection will depend on the crankshaft speed and the position of the lever of the all-range regulator of the fuel pump at the current time. The design also contains STA 471A chip, which is a key block. When the input signal from chip "NE555", the key block closes its output to the body, thereby providing a voltage supply to the nozzles. The key inputs are combined together, so when the input signal is applied, all nozzles open at the same time. The LED lights up when the nozzles open, thus displaying the injection intensity.

The pulse rate depends on the nominal values of the external capacitor and two resistances and is determined by the following formula (1):

$$
f=\frac{1}{0.693 \cdot C \cdot\left(R_{1}+R_{2}\right)}
$$

where $R_{l}$ and $R_{2}$ - external resistances of the time-setting circuit, $\mathrm{kOhm}$;

$\mathrm{C}$ - capacity of the external capacitor of the time-setting, $\mathrm{pF}$.

The time interval between the beginning of each next pulse is called the period and is indicated by letter T. It consists of the duration of the pulse itself $-T_{1}$ and the interval between pulses $-T_{2}$.

$T_{1}=0.693 \cdot\left(R_{1}+R_{2}\right) \cdot C$,

$T_{2}=0.693 \cdot R_{2} \cdot C$,

where $T_{l}$ is the pulse duration time, $\mathrm{s}$;

$T_{2}$ is the interval between pulses, $\mathrm{s}$.

Crankshaft position sensor 1 of the inductive type is designed to synchronize the work of the electronic control unit (ECU) with the top dead center of pistons 1 and 4 of cylinders and the angular position of the crankshaft. The master disc is a gear wheel with marks attached to its crown to create a synchronization pulse, which is necessary to coordinate the ECU operation with the engine operating mode.

The device works as it follows. The EG from the diesel engine comes from exhaust manifold 4 (Fig. 1,2) into the inlet of aerosol chamber 20. Passing through conical swirl 21, the gas flow acquires a directional rotating motion. The use of swirl 21 in the catalyst leads to the alignment of the local exhaust gas speeds and reduction in the uneven distribution of the flow velocities, which is very important for EGs cleaning process. Then the vortex flow is treated with water aerosol by nozzles 7 , installed radially in the housing of aerosol chamber 5. Nozzle 7 is a solenoid valve that allows the neutralizing solution to flow when voltage is applied to it and locks under the action of a return spring when the voltage is removed. The injection of aerosols is carried out with the frequency of the engine operation and is regulated by electronic control unit 19 .Simultaneous injection of the emulsion by at least three nozzles 7 imparts an additional rotational impulse to the moving stream. The processes of trapping, chemical bonding and neutralization of toxic components and soot particles contained in the EGs are carried out with direct contact between the exhaust gases being processed and 
the smallest drops of the neutralizing solution sprayed by nozzles 7 of aerosol chamber 5, whereby the developed surface of their contact is achieved, which allows the specified change of the state of the EG in the volume limited by aerosol chamber 5, over a short period of time. The process of sedimentation of soot particles and harmful substances on liquid droplets is determined by the mass of the liquid, the developed surface of droplets and the high velocity of the motion of particles of liquid and soot in the housing and the outlet of the aerosol chamber having a confusor shape. The efficiency of deposition largely depends on the uniform distribution of the fluid supplied by nozzles 7 over the cross section of aerosol chamber 5 .

After aerosol treatment, exhaust gases are removed from aerosol chamber 5 through outlet 22 and, passing through ejector 10 and mixing with atmospheric air, enter inlet 23 of swirl drop separator 11, where passing through conical swirl 24, the two-phase gasliquid flow acquires a directional rotary motion, while the liquid phase and foreign non-gaseous impurities that it has caught, under the action of centrifugal forces, are separated on the inner wall of swirl drop separator 11, and steam and the cleaned gas enter outlet of the centrifugal swirl drop separator 25 . The liquid phase, in the form of a thin wall film, moves through swirl drop separator 11 and enters the cavity between outlet of the centrifugal swirl drop separator 25 and swirl drop separator 11 and is removed through fluid removal pipe 26 . The cleaned gaseous state is moved out through outlet of the centrifugal swirl drop separator 25 to the atmosphere. Thus, swirl drop separator 11 contributes not only to the removal of harmful substances trapped by the solution, but also to a decrease in the amount of moisture carried to the atmosphere as a whole.

ECU 19 functions as it follows. When power supply to electronic control unit 19, integral timer unit 18 begins to generate an electrical signal of predetermined duration, depending on the nominal elements of time-setting circuits 2, 17, external resistors and capacitors. Circuit 17 is put into operation only when the absence or malfunction of electromagnetic crankshaft position sensor 1 of the engine connected to electronic control unit 19 and installed on the engine block. Integral timer unit 18 of ECU 19 generates signals based on the reference pulses received from crankshaft position sensor 1 , which are generated when passing a tag attached to the crown of the flywheel of the crankshaft. The basic impulse enters the input comparator of integral timer unit 18 . Then the IC chip-timer, being its main element, generates a periodic pulse, which, passing through resistor assembly 14, enters the input of the chip of key block 13.Key block 13, when a signal arrives, closes its outputs to the case, thereby opening the transit for the current from power supply 16, supplying the magnet windings of nozzles 7 . When the signal finishes, key block 13 opens its output with the case and stops the power of the nozzle windings until the next pulse. At the same time, with the supply voltage to electronic control unit 19 , the supply voltage goes to electric rotary liquid pump 9 , installed in tank with a neutralizing solution 8 , providing pressure of at least $300 \mathrm{kPa}$. Liquid pump 9 is connected to nozzles 7 by oil-resistant hoses 3 , by means of which the neutralizing solution is continuously supplied to nozzles 7 and then to the internal cavity of aerosol chamber 5, where it interacts with the exhaust gas. The neutralizing solution must be supplied to the aerosol chamber in a fine spray, with far penetrating ability of the smallest droplets flying in the exhaust gas stream. To do this, it must be supplied under high pressure. To compensate for the insufficient degree of EG purification, when the engine is operating at high load, time-setting circuit 2, which determines the time the nozzle is open, contains potentiometric regulator position sensor 6 of the fuel pump. The slider of sensor 6 connected to the main lever of the regulator (not shown in the diagram), when it moves, in the case of overload, changes the resistance of sensor 6 and entire time--setting circuit 2 , thereby integral timer unit 18 increases the time when nozzle 7 is open and increases the amount of neutralizing solution [21] supplied per unit time.
To improve the EG cleaning performance when nominal and increased load, time-setting circuit 17 (Fig. 4), determining the time of the nozzle being open, contains EG temperature gauge block 13 installed in the case of exhaust manifold 12 of swirl drop separator 10. EG temperature gauge block 13 is connected to the input of time-setting circuit 17 by means of operational amplifier 16 and block of current driver 14. When the temperature of the cleaned exhaust gases passing through exhaust manifold 12 and contacting temperature gauge block 13 , which may be due to inadequacy of the neutralizing solution going to aerosol chamber 5 to the EG. Temperature gauge block 13 changes the voltage at its terminals. Operational amplifier 16 amplifies the signal from temperature gauge block 13. Then its output voltage is converted into current with the help of block of current driver 14. The output current from block of current driver 14 goes to the input of timesetting circuit 17 , which determines the time of nozzles being open. Depending on the current magnitude in time-setting circuit 17, timer unit 3 changes the time when nozzles 7 are open and changes the amount of the neutralizing solution coming in a unit of time. Thus, there is an automatic regulation of the device operation mode, due to the appearance of temperature feedback in the system [22].

The thermoelectric method of temperature measuring is based on the occurrence of EMF) in a circuit composed of dissimilar conductors, when the thermal misbalance caused by the inequality of temperatures at the junction of the conductors. The emergence of thermo-EMF is due to the movement of electrons from one conductor to another and the alignment of their internal potentials [27].

The main element of EG temperature gauge block 13 is a thermal transmitting chip with a linear dependence of the output voltage on the temperature, type K1019EM1.

The sensor is similar in properties to a Zener diode with low differential resistance and with a stable and normalized positive temperature coefficient of voltage (TCV). The operation of the K1019EM1 temperature sensor is based on the temperature dependence of the difference in the voltage values at the emitter junction $\mathrm{U}_{\text {бэ }}$ of two transistors with different emitter current density. In order to improve the accuracy of temperature measurement in the chip, a calibration circuit is provided.

Pressure increases in the intake manifold of swirl drop separator 10 , having the form of a diffuser, and the flow rate decreases, which contributes to coagulation of small particles.

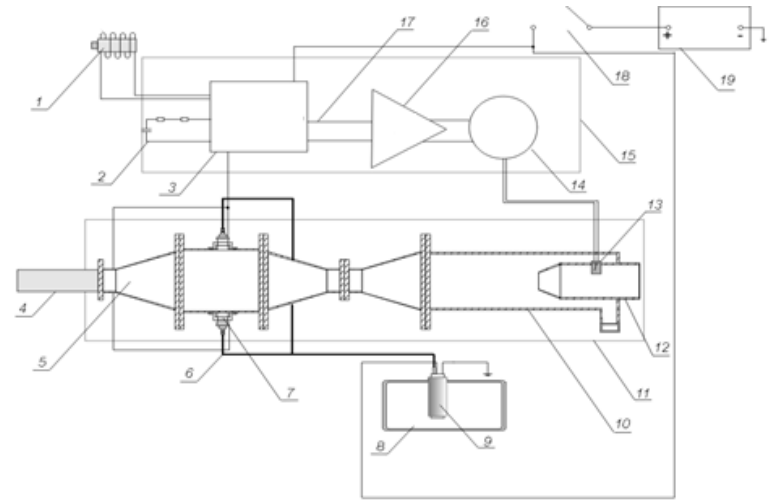

1- crankshaftpositionsensor; 2, 17 - time-settingcircuits; 3 - timerunit; 4 exhaustmanifold; 5 - aerosolchamber; 6 - solutionsupplyhoses; 7 nozzles; 8 - tankwithaneutralizingsolution; 9 - liquidpipe; 10 swirldropseparator; 11 - liquidpipe; 12 - exhaustmanifold; 13 temperaturegaugeblock; 14 - block of current driver; 15 - electronic control unit, 16 - operational amplifier; 18 - key; 19 - power supply.

Figure 4: Diagram of the device for cleaning exhaust gases of diesel internal combustion engines with a temperature gauge block.

There is no ejector device in this variant of the construction for EG cleaning, as the atmospheric air drawn in by it and mixed with EG sharply reduces the information content and, as a result, the 
efficiency of the temperature gauge block, due to some additional decrease in their temperature.

\section{Results}

To evaluate a number of power and economic parameters of a diesel engine, a series of experiments was carried out. In order to obtain minimum errors, studies of the process of cleaning exhaust gases of the tractor were carried out under conditions as close as possible to the operating conditions. According to the results of the investigations, some graphical dependencies were obtained that determine the power and economic performance of the diesel engine of a tractor with a standard and modernized exhaust system. These results are presented in the following figures.

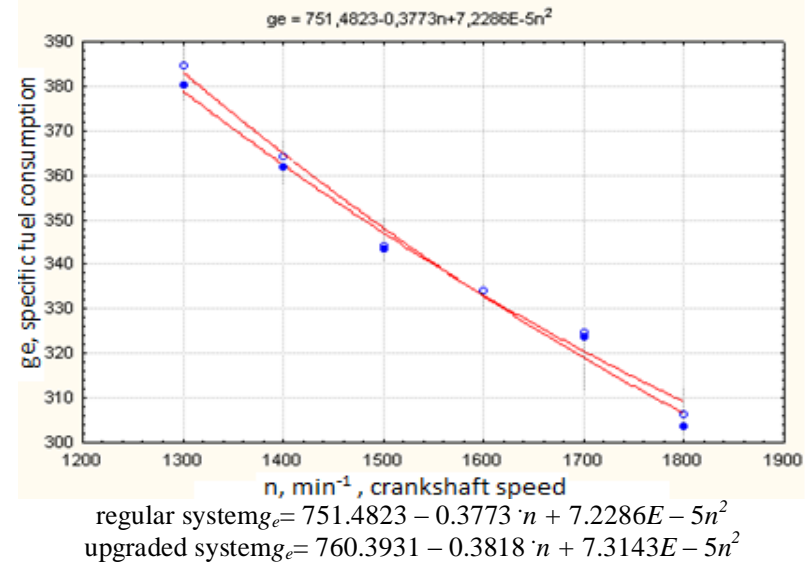

Figure 5 - Graphic dependence of the change in specific fuel consumption $g_{e}$ on the change in the rotational speed of the engine crankshaft when working with regular and modernized exhaust systems.

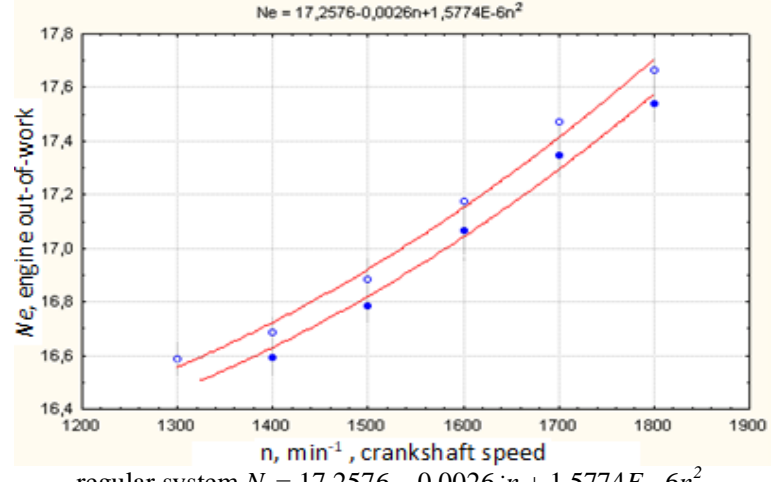

regular system $N_{e}=17.2576-0.0026 \cdot n+1.5774 E-6 n^{2}$ upgraded system $N_{e}=17.5829-0.0026 \cdot n+1.067 E-6 n^{2}$

Figure 6 - Graphic dependence of the change in the engine out-of-work of tractor T-30-69 $\mathrm{N}_{\mathrm{e}}$, on the change in the rotational speed of the crankshaft when working with the regular and upgraded exhaust systems.

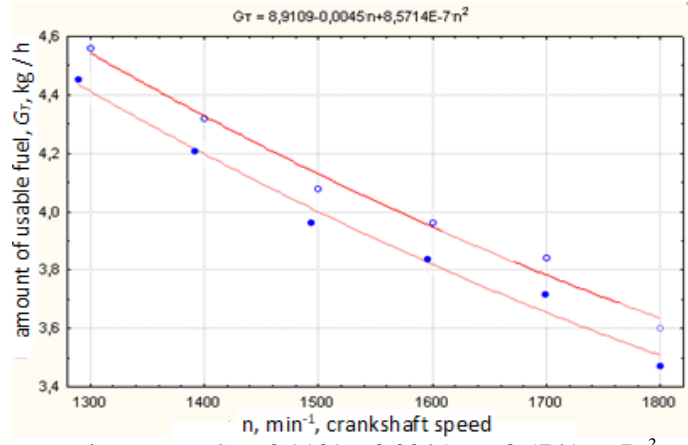

regular system $G_{T}=8.9109-0.0045 \cdot n+8.5741 E-7 n^{2}$

upgraded system $G_{T}=8.0891-0.0046 n+8.7429 E-7 n^{2}$

Figure 7 - Graphic dependence of the change in the amount of usable fuel $\mathrm{G}_{\mathrm{T}}$ on the change in the crankshaft speed when working with regular and upgraded exhaust systems.
Having analyzed the presented graphical dependencies, it is possible to conclude that the introduction into the tractor engine exhaust system will not have any significant impact on its power and fuel-economic parameters. At the same time, the out-of-work decrease of the engine, equipped with an upgraded exhaust system when taking the external speed characteristic averaged $1.6 \%$, the torque was $1.5 \%$ and the increase in specific fuel consumption was $1.8 \%$.

To establish the cumulative effect of the amount of cold steam and the duration of cold steam supply on the toxicity and opacity of exhaust gases of the diesel engine, a two-factor experiment was conducted.

At the first stage of laboratory investigations, when taking down the characteristics of the engine in the mode of free acceleration, the dependent factor was the duration of spraying the neutralizing solution, which determines the change in the EG opacity.

As a result of statistical processing of experimental data, a mathematical model was obtained, which determines the dependence of the EG opacity on the duration of spraying the neutralizing solution into the aerosol chamber of the catalyst.

To compensate for the insufficient degree of EG purification, when the engine is operating in high load mode, the time-setting circuit of the electronic control block, which determines the time the injector is open, contains a sensor for the fuel pump regulator position.

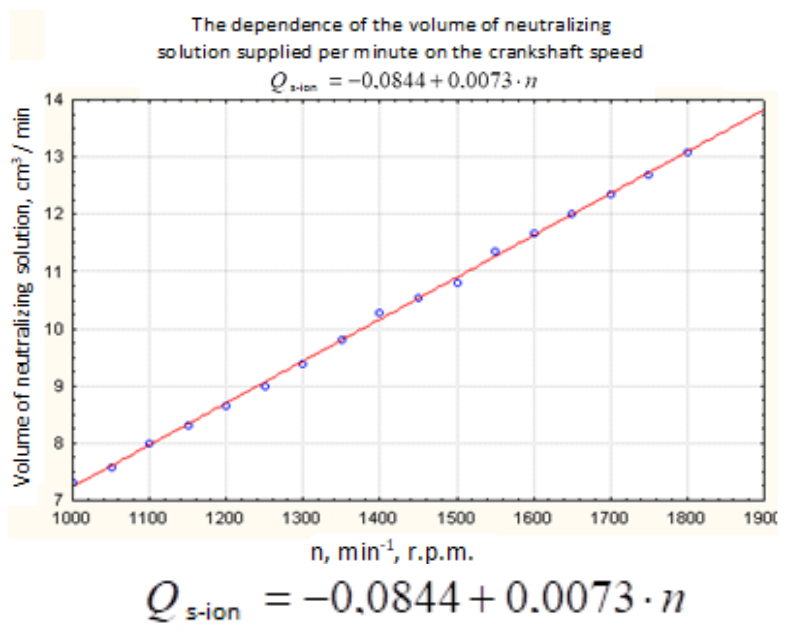

Figure 8 - Graphic dependence of the neutralizing solution flow rate on the engine crankshaft speed with an average feed time of $15 \mathrm{~ms}$.

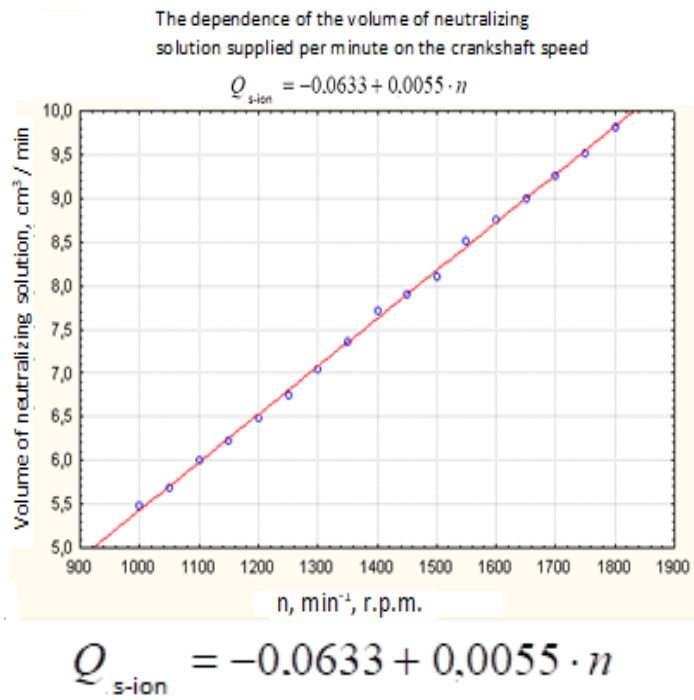

Figure 9 - Graphic dependence of the volume of neutralizing solution supplied per minute on the crankshaft speed at an average feed time of 20 $\mathrm{ms}$ 


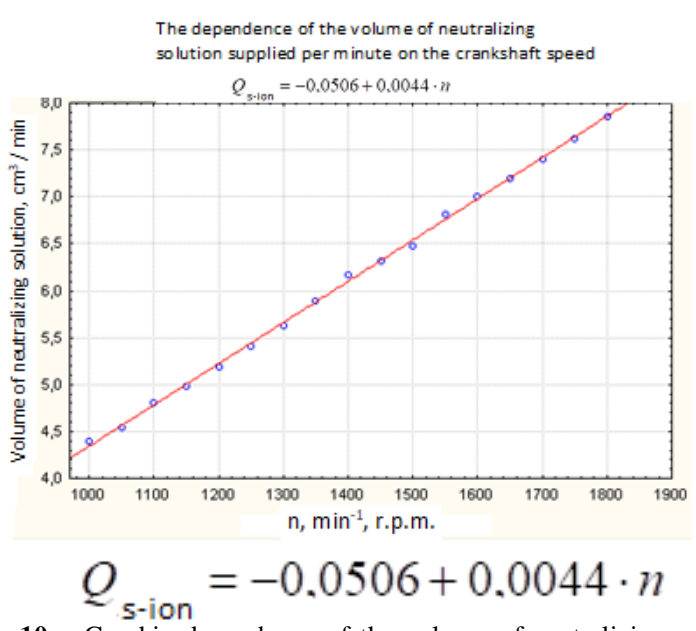

Figure 10 - Graphic dependence of the volume of neutralizing solution supplied per minute on the crankshaft speed at an average feed time of 25 ms.



Figure11:Graphicdependenceofthevolumeofneutralizingsolutionsuppliedp erminuteonthecrankshaftspeedatanaveragefeedtime of $30 \mathrm{~ms}$.

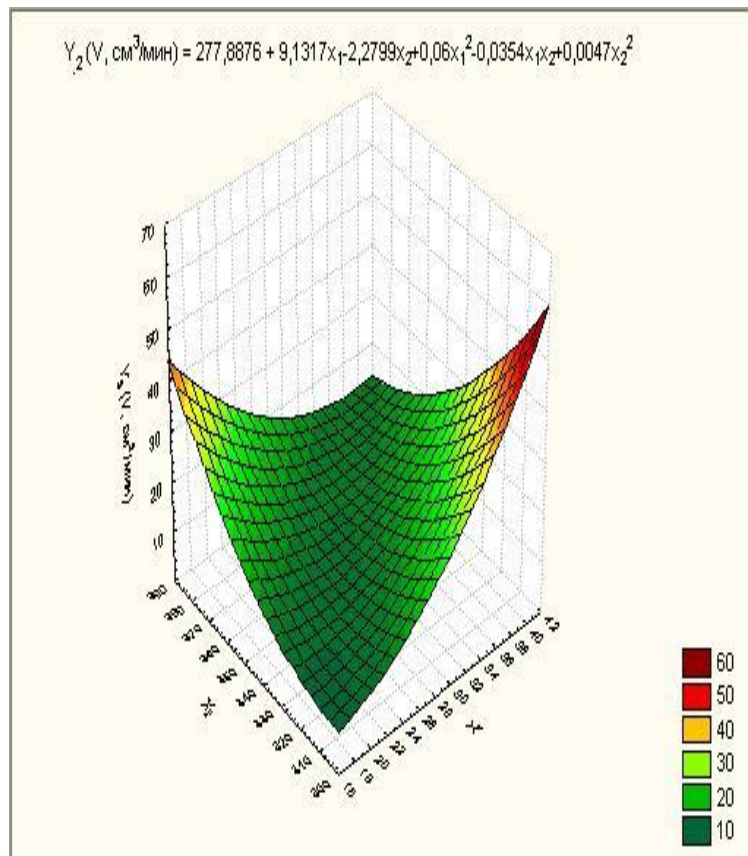

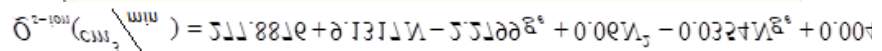

Figure 12: Graphic dependence of the neutralizing solution flow rate on fuel consumption and the degree of EG purification from carbon monoxide.

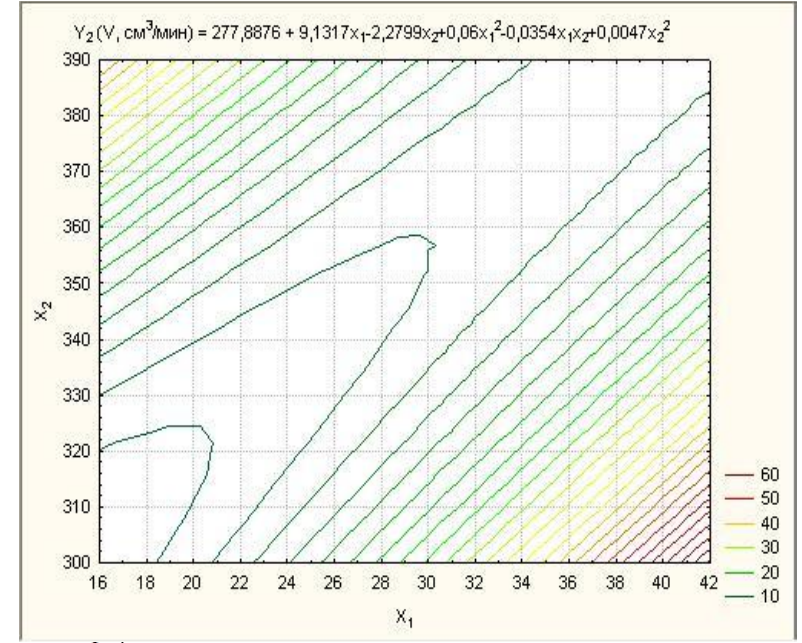

$Q_{s-\text { ion }}\left(\mathrm{cm}^{3} / \min \right)=277.8876+9.1317 N-2.2799 g_{\varepsilon}+0.06 N^{2}-0.0354 N g_{\varepsilon}+0.0047 g_{\varepsilon}^{2}$

Figure 13: Graphic dependence of the neutralizing solution flow rate on fuel consumption and the degree of EG purification from carbon monoxide.

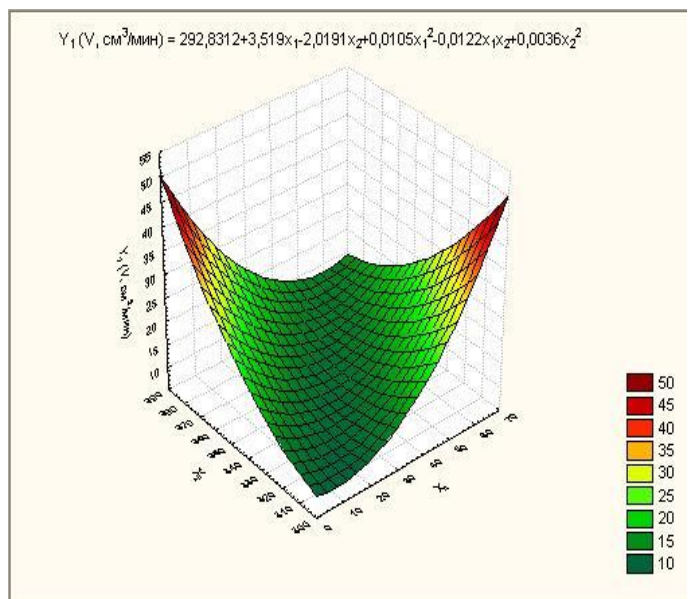

$Q_{i-\operatorname{ion}}\left(\mathrm{cm}^{3} / \min \right)=292.8312+3.519 \mathrm{~N}-2.0191 g_{\theta}+0.0105 \mathrm{~N}^{2}-0.0122 \mathrm{Ng}+0.0036 g_{\theta}^{2}$

Figure 14: Graphic dependence of the neutralizing solution flow rate on fuel consumption and the degree of EG purification from nitrogen oxides.

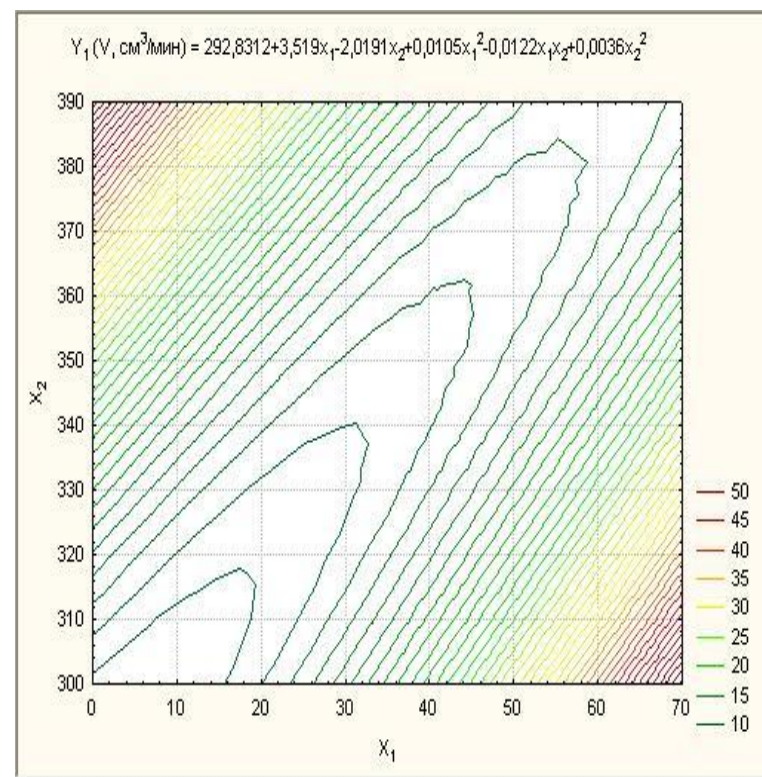

$Q_{i-1002}\left(\mathrm{~cm}^{3} / \mathrm{min}\right)=292.8312+3.519 \mathrm{~N}-2.0191 g_{i}+0.0105 \mathrm{~N}^{2}-0.0122 \mathrm{Ng} g_{e}+0.0036 g_{g}^{2}$

Figure 15: Graphic dependence of the neutralizing solution flow rate on fuel consumption and the degree of EG purification from nitrogen oxides. 


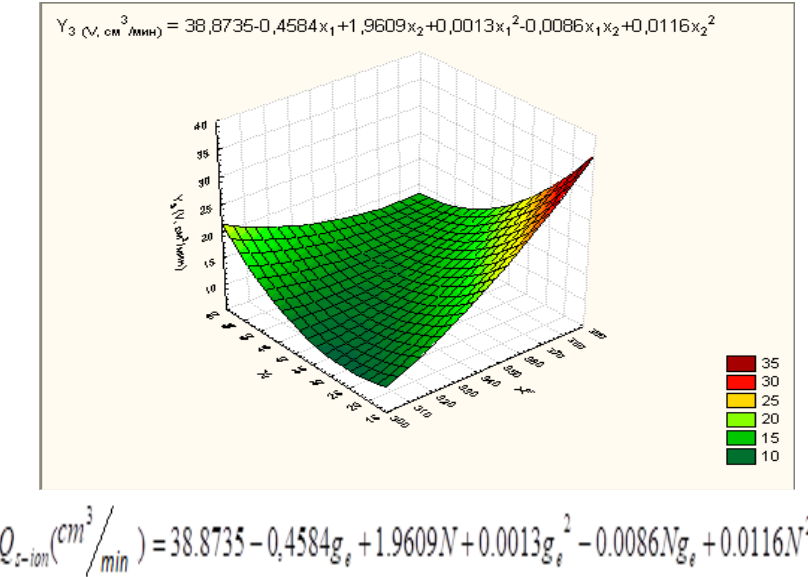

Figure 16: Graphic dependence of the consumption of neutralizing solution (cold steam) on fuel consumption and the degree of EG purification from soot.

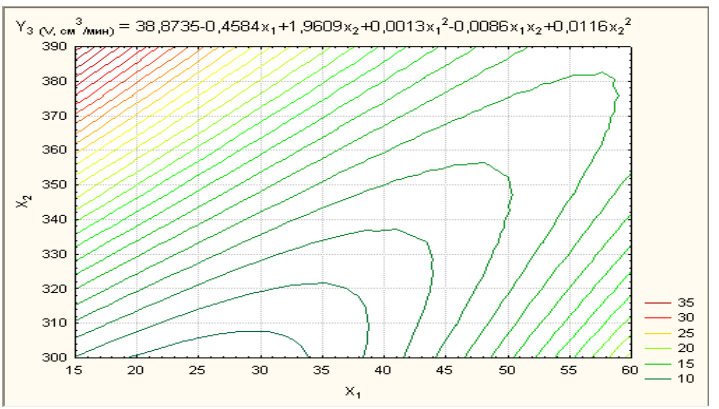

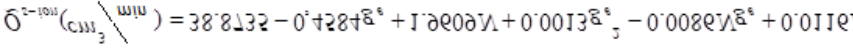

Figure 17: Graphic dependence of the neutralizing solution flow rate on fuel consumption and the degree of EG purification from soot.

$Y_{t}\left(V_{1}, \mathrm{~cm}^{3} \mathrm{MnH}\right)=298,932+4,0726 x_{1}-2,1681 x_{2}+0,0127 x_{1}^{2}-0,0146 x_{1} \times x_{2}+0,004 x_{2}^{2}$
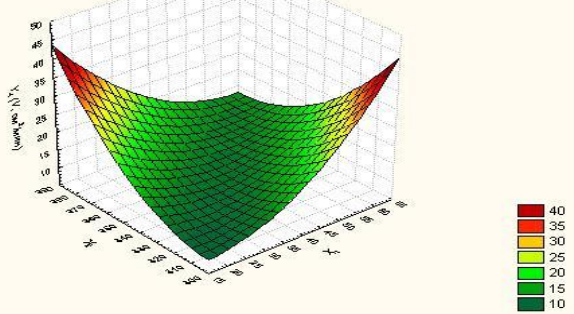

$Q_{\text {s- } 10 \mathrm{~m}}\left(\mathrm{~cm}^{3} / \min \right)=298.932+4.0726 \mathrm{~N}-2.1681 \mathrm{~g}_{\ell}+0.0127 \mathrm{~N}^{2}-0.0146 \mathrm{Ng}_{\ell}+0.004 \mathrm{~g}_{\ell}^{2}$

Figure 18 - Graphic dependence of the neutralizing solution flow rate on fuel consumption and the degree of EG purification from hydrocarbons.

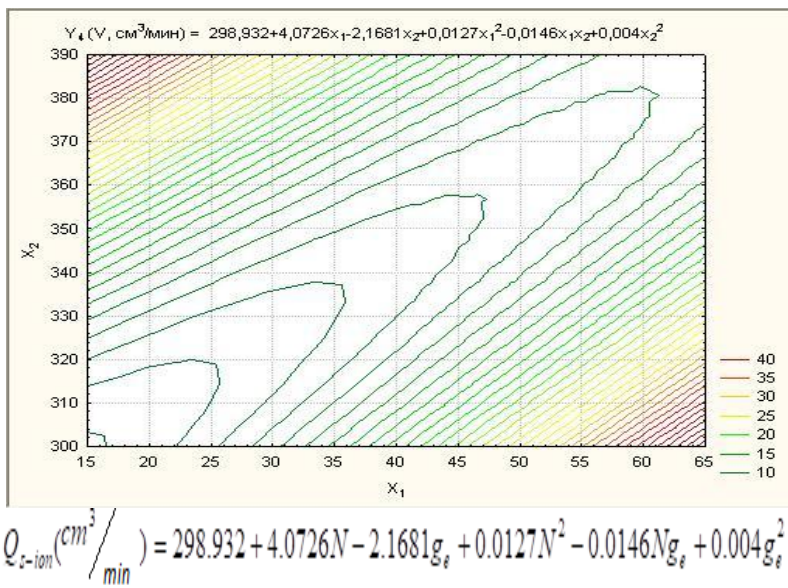

Figure 19 - Graphic dependence of the neutralizing solution flow rate on fuel consumption and the degree of EG purification from hydrocarbons.
Thus, in the framework of the experiment, it was necessary to establish regularities of changes in concentrations of toxic components in the EG composition depending on the amount of the neutralizing solution supplied for the subsequent fine-tuning of the automated control system of the liquid catalyst to achieve maximum cleaning performance.

From the given dependences it is clear that members of higher orders do not have any significant effect on the model because of small values of coefficients and the dependencies can be regarded as linear.

Processing the obtained data was carried out in accordance with the above methodology by statistical programs "Statistika" and "Microsoft Excel" using a PC IBM PC.

\section{Discussion}

It has been found that one of the most important properties of aerosols in general, and soot in particular, is the continuous and spontaneous coagulation of their particles. The particles of a substance in contact merge or stick together and the aerosol becomes more and more coarse. Contact occurs as a result of the particles movement, which leads to their connection with each other and thus reduction of the total number of individual particles. The carbon atoms located on the edges of the crystal lattices have free valences, along which atoms of individual flat carbon lattices or whole chains of atoms join them. It is well known that soot particles, due to their structure and significant specific surface, absorb from the gas stream and adsorb on their surface some harmful substances. These are the cancerogenic polycyclic hydrocarbon benz- $\square$-pyren, formed in the combustion chamber during the pyrolysis of fuel, in parallel with soot formation, as well asdeins- $\square$ j-pyren, debine-( $\square$,h)-athrencen, cyclopentene-(c,d)-pyren, chrysene, benz-( $\square$ )-athrencen, aniraktren and others.

Thus, it can be concluded that soot particles are capable of carrying off on their surface a certain amount of harmful components from the engine exhaust gases. By removing the soot particles from the exhaust gas flow, while increasing the share of adsorption of harmful components of exhaust gases on them, we have an additional opportunity to reduce the amount of harmful components entering the environment when diesel engines function.

It is possible to increase the share of adsorption of harmful components of exhaust gases on the surface of soot particles in several ways, for example, by creating conditions for controlled turbulization of the flow with the help of some special devices swirls. Swirling flow has a number of advantages over direct flow. It is an intensive turbulent exchange and the presence of recycling zones, contributing to stabilization of chemical processes and intensive mass transfer between substances [11].Moving in a swirling flow, the soot particles will, firstly, come into contact with each other more often, which will lead to their coagulation and consolidation into larger conglomerates, and secondly, the particles will be able to adsorb more molecules of harmful substances from exhaust gases. Coagulation has some positive effect on soot trapping processes, because, due to the high degree of dispersion of diesel soot and its relatively low concentration in exhaust gases, the efficiency of using such widely used and wellestablished devices, like multicyclones, on some engine operating modes does not exceed $60 \%$, and this is when significant increase in back pressure at exhaust [28].

It should be noted that apparatuses for dry cleaning of gases, which are based on the effect of inertia forces, gravitational or centrifugal forces acting on a suspended particle, are relatively simple in design, inexpensive to manufacture and maintain, and do not require additional devices for performing the working process in comparison to electro filters used for gas cleaning.

Studies show that it is possible to increase the adsorption of harmful components by soot particles by using some chemicals. In 
particular, studies [14] show that nitric acid is physically adsorbed on soot and its adsorption increases with increasing the amount of water participating in the reaction. Thus, toxic nitrogen oxides, having entered into a chemical reaction with water molecules and turning into a new substance, nitric acid, are more intensively adsorbed on the surfaces of soot particles, and some of the nitric acid molecules can react with them.

From the foregoing it follows that some physical processes occurring in the apparatus for dry cleaning of exhaust gases, when combined with chemical processes that take place during wet cleaning of exhaust gases, can improve the quality of cleaning, due to the optimal use of the properties of substances involved in the process [22].

\section{Conclusion}

For example, in the process of mechanized works performed by a tractor in greenhouse blocks, the concentrations of toxic substances contained in the exhaust engine of a diesel engine significantly increase. So in 2 hours after the tractor starts working, they exceed the maximum allowable concentrations of soot by 12.5 times, benz- $\square$-pyren by 8.93 times and hydrocarbons by 6.83 times, which makes it difficult to use the tractor inside the greenhouses, especially in the autumn-winter period. When natural ventilation functions the concentration of toxic substances after two hours of the tractor operation will reach the maximum allowable values for soot in 6 hours, for benz- $\square$-pyren and hydrocarbons in 4 hours.

When mechanized work in the greenhouse, a method of wet cleaning of exhaust gas in the LC was used. The method consists in supplying a portion of finely dispersed neutralizing solution to the irrigation of a turbulent EG stream followed by separation of the neutralizing solution and harmful substances, which significantly reduces exhaust emissions. The solution consisting of $85 \%$ of water $\mathrm{H}_{2} \mathrm{O}$ was chosen as a neutralizing substance. Its composition included: an aqueous solution of soda $\mathrm{Na}_{2} \mathrm{CO}_{3}$ in the amount of $14.5 \%$ by volume and $0.5 \%$ of 1.4-dihydroxybenzene (hydroquinone) $\mathrm{C}_{6} \mathrm{H}_{4}(\mathrm{OH})_{2}$.

A constructive-technological scheme of the device for cleaning the exhaust gas of a diesel internal combustion engine has been developed. Itincludesa liquid catalyst consisting of an aerosol chamber, a swirl drop separator and an ejector, an electronic control unit, nozzles for supplying a neutralizing solution, a crankshaft position sensor and a sensor controlling the position of the regulator of the all-mode fuel injection pump assembly, a temperature gauge block with an operational amplifier and a block of current driver of the liquid pump.

It is theoretically proved that the process of neutralizing the toxic components of the diesel engine exhaust gas in the liquid catalyst is determined by a number of factors, such as efficient irrigation of the exhaust gas by the neutralizing solution, coagulation of the neutralizing solution droplets and soot particles into larger formations and separation of the neutralizing solution droplets, toxic components and soot particles. In addition, the relationship between the flow rate of the neutralizing solution, the amount of exhaust gas, the amount and composition of the fuel supplied to cylinders has been theoretically proved.

The proposed method and the developed EG cleaning system of a tractor diesel engine can significantly reduce the concentrations of toxic substances in the air, as compared to the standard tractor exhaust system. The system reduces the amount of hydrocarbons by $14 \%$, benz- $\square$-pyrenby $18 \%$ and soot by $22.4 \%$. In the composition of exhaust gases, concentrations of toxic substances are reduced by $40 \%$ for oxides of nitrogen, by $43 \%$ for hydrocarbons, by $70 \%$ for soot, and the noise level of engine operation is reduced by $16-22 \%$.

Calculations of economic efficiency have shown that the use of the developed cleaning system allows to reduce the overall economic damage to the health of the staff by 279,118 rubles per year per tractor.

The economic effect from the use of a single tractor with an upgraded exhaust system of the diesel engine is 175,481 rubles / year with a payback period of about half a year.

\section{References}

[1] Janulevičius A., Juostas A. andČiplienè A.(2017). 'Nitrogen-oxide emissions from diesel-engine farm tractors during real-life cycles and their correlation with the not-to-exceed operating zones', Biosystems Engineering, Vol. 161, September, pp. 93-105.

[2] Pham E.J.M., Zhao D., Deng Y., Le D., Zuo W., Zhu H., (...), Zhang Z. (2017). 'Effect of different technologies on combustion and emissions of the diesel engine fueled with biodiesel: A review', Renewableand Sustainable Energy Reviews, 80, pp. 620-647.

[3] Bacenetti J., Lovarelli D., Facchinetti D., Pessina D. (2018). 'An environmental comparison of techniques to reduce pollutants emissions related to agricultural tractors', Biosystems Engineering, 171, pp. 30-40.

[4] Backman J., Piirainen P. and Oksanen T. (2015). 'Smooth turning path generation for agricultural vehicles in headlands', Biosystems Engineering, 139, pp. 76-86.

[5] Bietresato M., Calcante A. and Mazzetto F. (2015). 'A neural network approach for indirectly estimating farm tractors engine performances', Fuel, 143, pp. 144-154.

[6] Damanauskas V. and Janulevičius A. (2015). 'Differences in tractor performance parameters between single-wheel 4WD and dualwheel 2WD driving systems', Journal of Terramechanics, 60, art. no. 628, pp. 63-73.

[7] Elsanusi O.A., Roy M.M. and Sidhu M.S. (2017). 'Experimental Investigation on a Diesel Engine Fueled by Diesel-Biodiesel Blends and their Emulsions at Various Engine Operating Conditions', Applied Energy, 203, pp. 582-593.

[8] Gonzalez-de-Soto M., Emmi L., Benavides C., Garcia I. and Gonzalez-de-Santos P. (2016). 'Reducing air pollution with hybrid-powered robotic tractors for precision agriculture', Biosystems Engineering, 143, pp. 79-94.

[9] Graf, Plessen M.M., Bemporad A. (2017). 'Reference trajectory planning under constraints and path tracking using linear timevarying model predictive control for agricultural machines', Biosystems Engineering, 153, pp. 28-41.

[10] Grafkina M.V., Azarov A.V., Dobrinsky D.R., Tihonova M.M. Negative effects analysis for ecosystem elements impact of dust emissions from passing motor vehicles at different road coating types // Modern Science Success. 2016. Volume 8. № 12. P. 142147.

[11] Janulevičius A., Juostas A. and Čipliene A. (2016). 'Estimation of carbon-oxide emissions of tractors during operation and correlation with the not-to-exceed zone', Biosystems Engineering, 147, pp. 117-129.

[12] Gievoy S.A. (2003). 'Reduction of harmful emissions during operation of automotive diesel engines through the use of a diesel particulate filter', dis. cand. of tech. sc., M., RSL.

[13] Erokhin A.V. (2004). 'Technology and system for removal of exhaust gases from the internal combustion engines of a tractor with an ejector device to reduce their temperature', dis. cand. of tech. sc., Ryazan, RSHA.

[14] Lebedev P.T. (1973). 'The microclimate of premises for animals and the methods of its research', M., Rosselkhozizdat, $128 \mathrm{p}$.

[15] Levanchuk A.V. Hygienic characteristics of the environment pollution with products of operational wear of the braking system of cars // Modern Science Success. 2016. Volume 3. № 2. C 82-85.

[16] Lukhovitskaya E.E. (2007). 'Mass-spectrometric study of heterogeneous processes with the participation of soot, characteristic of the upper troposphere', abstract of dis. cand. of tech. sc., Moscow, Institute of Chemical Physics, RAS.

[17] Maksimenko O.O. (2006). 'Technology for improving the state of the air in restricted areas when operating internal combustion engines with liquid neutralizers in them', dis. cand. tech. sc., Ryazan, RSAA, $181 \mathrm{p}$.

[18] Markelova S.V., Prokhorova L.M. Ensuring ecological safety of the region as a criterion of sustainable development of the region // Modern Science Success. 2016. Volume 2. № 9. P. 67-73.

[19] Oleynik D.O. (2009). 'Catalyst for cleaning exhaust gases of diesel engines', Monthly Sc. J. "Young Scientist", no. 5, pp. 9-13. 
[20] Oleynik D.O. (2009). 'Device for cleaning exhaust gases of diesel engines with automatic regulation of the mode of operation', Monthly Sc. J. "Young Scientist", no. 8, pp. 17-20.

[21] Oleynik D.O., Trishkin I.B., Generalov V.S. (2008). 'Vocational health passport of an employee of the agro-industrial complex', Bulletin of Moscow State Agr. Engin. Univ., no. 2 (27).

[22] Oleynik D.O. and Generalov V.S. (2007). "'Health Passport" of an employee of the agro-industrial complex', Proceed. of the III Intern. Sc. and Pract. Conf. "European Scientific Space - 2007", vol. 5, Biol. Sc., Chemistry and chem. tech., Ecology. Agric. Vet. Med., Dnepropetrovsk, Science and Education, $104 \mathrm{p}$.

[23] Pat. 77353 Russian Federation, IPC F01N 3/02. 'Device for cleaning exhaust gases of internal combustion engines', I.B. Trishkin, D.O. Oleynik, Applicant and patent holder of FSEI HPE RSAA (RU), no. 2008115609/22; declare 04/21/2008; publ. 10/20/2008, Byul. no. 29, 2 p.

[24] Pat. 83292 Russian Federation, IPC F01N 3/02. 'Device for cleaning exhaust gases of internal combustion engines', Trishkin I.B., Oleynik D.O., Applicant and patent holder of FSEI HPE RSAA (RU), no. 2008148586/22, declare 09.12.2008, publ. 27.05.2009, Byul. no. 15, 2 p.

[25] Pat. 86665 Russian Federation, IPC F01N 3/02. 'Device for cleaning exhaust gases of internal combustion engines', Trishkin I.B., Oleynik D.O., Applicant and patent holder of FSEI HPE RSAA (RU), no. 2009113715/22; declare 04/14/2009; publ. 10.09.2009, Bull. no. 25, 2 p.

[26] Rakhmanin Yu.A., Levanchuk A.V. Qualitative and quantitative characterization of environmental pollution by products of operational wear of components of the road-car complex // Modern Science Success. 2016. Volume 1. № 4. P. 158-162.

[27] Strazhev N.P. (2007). 'Method and device for reducing smoke and noise level in rooms when diesel engines operate in them', dis. cand. tech. sc., Ryazan, RSAA, 205 p.

[28] Trishkin I.B. (2000). 'Method and device for reducing the toxicity of a tractor diesel engine when performing mechanized work in greenhouses', dis. cand. tech. sc., Ryazan, RSAA.

[29] Trishkin I.B. and Oleynik D.O. (2009). 'A device for cleaning exhaust gases of internal combustion engines', Bul. of FSEI HPE "Moscow State Agro-Engineering University", no. 1 (32), pp. 6668.

[30] Trishkin I.B. (2009). 'Constructive-technological scheme of the device for cleaning exhaust gases of diesel internal combustion engines', Monthly Sc. J. "Young Scientist", no. 9, pp. 17-21.

[31] Chistyakov S.F. and Radun D.V. (1972). 'Thermal measurements and devices: studies. manual for universities', M., High School, 392 p.

[32] Yushin V.V., Popov V.M., Kukin P.P. et al. (2005). 'Technique and technology of environmental protection: studies. manual for universities', M., Higher school, 391 p. 